\title{
Cerebral Ischemia Promotes Rich Pseudopalisading Necrosis in the Rat C6 Glioblastoma Model
}

\author{
Mahabub H. MAmun, Hideki KAmitani*, Yusuke KinOSHITA, \\ Sadaharu TABUCHI, Brian WASITA, and Takashi WATANABE \\ Department of Neurosurgery, Institute of Neurological Sciences, \\ Faculty of Medicine, Tottori University, Yonago, Tottori; \\ ${ }^{*}$ Department of Neurosurgery, Tottori Prefectural Kousei Hospital, Kurayoshi, Tottori
}

\begin{abstract}
The effect of hypoxia on glioma growth including pathological changes was investigated in an experimental model of brain ischemia in the rat C6 glioma model. C6 glioma cells were inoculated into the subcortex of adult Wistar rats. Focal cerebral ischemia near the implanted glioma area was induced by permanent middle cerebral artery occlusion (PMCAO). Ten days later, the rats were sacrificed to compare tumor volume of C6 glioma without PMCAO (control group) versus C6 glioma with PMCAO (hypoxia group). The histological features were also observed. The mean tumor volume in the hypoxia group was significantly larger than that in the control group. The most prominent histological finding in the hypoxia group was abundant formation of pseudopalisading around the necrotic areas. Immunohistological examinations showed intensive staining for vascular endothelial growth factor and hypoxia-inducible factor in these pseudopalisading cells. These findings suggest that cerebral ischemia positively modulates glioma mass growth by the formation of pseudopalisading necrosis, a characteristic histological finding of glioblastoma.
\end{abstract}

Key words: cerebral ischemia, hypoxia, glioblastoma, pseudopalisading, C6 glioma

\section{Introduction}

Hypoxia is a condition of low tissue oxygen tension associated with acute and chronic vascular diseases, and pulmonary disease. Hypoxia may also occur within malignant tumors due to the development of aberrant blood vessels resulting in poor blood flow. Cancer cells undergo genetic and adaptive changes under hypoxic conditions that allow survival and even proliferation in the hypoxic environment. ${ }^{1)}$ Such changes contribute to the malignant phenotype and aggressive tumor behavior. ${ }^{11)}$ In addition, brain tumor progression under hypoxic conditions is associated with induction of hypoxia-inducible factor (HIF)-1, ${ }^{12,23)}$ which up-regulates several angiogenesis factors including vascular endothelial growth factor (VEGF). ${ }^{6,22)}$

Glioblastoma has the most aggressive phenotype among primary human brain tumors, and exhibits extensive necrotic regions surrounded by hypercellular zones termed pseudopalisading. ${ }^{14)}$ The cells that form pseudopalisading are known to secrete

Received June 27, 2008; Accepted February 5, 2009 high levels of proangiogenic factors such as HIF- $1 \alpha$ and VEGF that promote tumor growth. ${ }^{3)}$ The hypoxic and pseudopalisading cells in glioblastoma exhibit enhanced gelatinase activity, which is typical for an invasive phenotype. ${ }^{4)}$ Therefore, glioblastoma formation may be linked to the presence of hypoxia. However, little is known about effect of ischemic brain on glioma growth in the clinical situation. Numerous in vitro studies have suggested that hypoxia can induce proliferation in malignant cells by activation of several genes including HIF- $1 \alpha$, and can accelerate tumor growth by neovascularization linked with VEGF.6,15)

The present study developed an experimental model of cerebral hypoxia in the rat based on induction of focal cerebral ischemia by permanent middle cerebral artery occlusion (PMCAO) method, ${ }^{15,16)}$ to investigate tumor growth and histological changes possibly involved in the formation of glioblastoma.

\section{Materials and Methods}

Thirty-nine Wistar rats (SLC, Inc., Sendai) weighing 250-300 g were used for this study. Rats were 


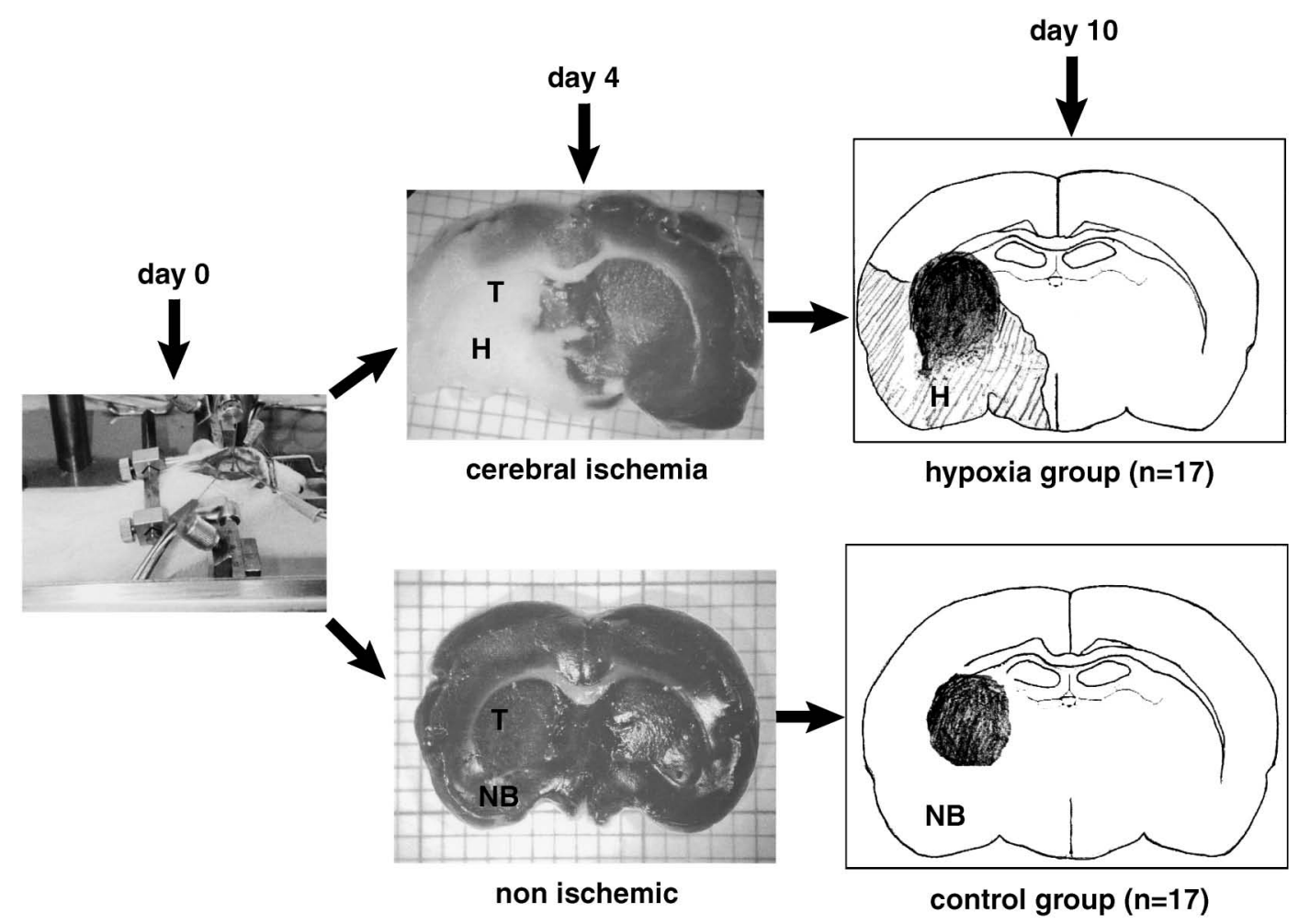

Fig. 1 Experimental design for induction of hypoxia in rat brain containing implanted C6 glioma. Cultured $\mathrm{C6}$ glioma cells $\left(2.5 \times \mathbf{1 0}^{4}\right)$ were implanted in the right subcortical area using a stereotaxic apparatus on day 0 . The right common carotid artery was permanently occluded on day 4, resulting in hypoxia in the right hemisphere of the hypoxia group, or only sham operation in the control group. Rats in both groups were sacrificed for analysis of the tumor volume and histological changes on day 10. H: hypoxia, NB: normal brain, T: tumor.

housed in cages and had free access to standard laboratory rat food and water. All experiments were performed with the approval of the Animal Care and Use Committee of Tottori University.

Rat glioma cell lines, C6 cells, were obtained from the Health Research Resource Bank (Osaka). This cell line was derived from N-methyl nitrosoureatransformed rat astrocytes. ${ }^{2)}$ The cells were cultured under sterile conditions at $37^{\circ} \mathrm{C}$ in Dulbecco's Modified Eagle's Medium (GIBCO BRL, Grand Island, N.Y., U.S.A.) supplemented with $10 \%$ fetal bovine serum (GIBCO BRL) and 1\% penicillin/streptomycin (GIBCO BRL) in a humidified incubator under $5 \%$ $\mathrm{CO}_{2}$ and $95 \%$ air. After the cultured cells became confluent, they were washed in phosphate buffered saline and harvested by the trypsin supplier. The cells were suspended to obtain a concentration of $2.5 \times 10^{4}$ cells per $1 \mu \mathrm{l}$ of the cultured medium in a tube for implantation.

Figure 1 shows an overview of the experimental procedures. A total of 39 rats were included in the two independent experiments. Two rats died due to anesthetic complications, one rat died during the
PMCAO surgery, and two rats died the day after PMCAO. Therefore, a total of 34 rats were injected with the C6 glioma cells, and then 4 days later underwent either PMCAO (hypoxia group, 17 rats) or sham PMCAO (control group, 17 rats). The total experimental duration was 10 days, after which all the rats were sacrificed.

The rats were anesthetized with intraperitoneal injection of pentobarbital $(45 \mathrm{mg} / \mathrm{kg})$, and then shaved and sterilized with $1 \%$ povidone-iodine. The head was mounted into a stereotactic frame (SR-6N; Narishige Scientific Instrument Lab., Tokyo) by consulting an anatomical map. ${ }^{7)}$ A burr hole was made in the right cranial bone. The brain cortex coordinates are described as the distances from the level of the interaural line ${ }^{18)}$ and the center of the sagittal sinus, as follows: anterior-posterior $-2.0 \mathrm{~mm}$, lateral $-4.5 \mathrm{~mm}$, height $-4.0 \mathrm{~mm}$. Intracerebral injection of $2.5 \times 10^{4}$ cells suspended in $1 \mu \mathrm{l}$ of culture medium was performed using a 5.0- $\mu$ l scale Hamilton syringe (Hamilton Company, Reno, Nev., U.S.A.) over approximately 20 minutes. ${ }^{10)}$

Four days after implantation of the C6 glioma 
cells, 17 rats were anesthetized with pentobarbital and underwent PMCAO to induce focal cerebral ischemia ${ }^{8,13)} \mathrm{A}$ midline incision was made above the right common carotid artery region. The internal carotid artery was isolated up to the carotid canal. Arteriectomy of the external carotid artery was performed and a (4-0) monofilament rounded tip suture was introduced into the external carotid artery stump. The middle cerebral artery was then occluded with an intraluminal suture. The microvascular clip was released from the common carotid artery and the artery was permanently ligated. The body temperature was continuously monitored with a rectal probe (Homeothermic Blanket Systems; Harvard Apparatus, Holliston, Mass., U.S.A.) and maintained at $37^{\circ} \mathrm{C}$ until the end of the surgical procedure. ${ }^{15)}$

Rats were sacrificed on day 10 after C6 glioma implantation. The brains were perfused with formalin overnight, then dehydrated and embedded in paraffin. Coronal sections of the brains $(4 \mu \mathrm{m})$ were cut, and stained with hematoxylin and eosin (H\&E), monoclonal mouse anti-Ki-67 (MIB-5) (Dako, Carpinteria, Calif., U.S.A.), rabbit polyclonal antibody antiHIF-1 $\alpha$ (Santa Cruz Biotechnology, Inc., Santa Cruz, Calif., U.S.A.), and rabbit polyclonal antibody antiVEGF (Santa Cruz Biotechnology, Inc.). Immunostaining was performed using the avidinbiotin-peroxidase method. Briefly, sections were incubated with the appropriate antibodies diluted with distilled water (1:100) for 1 hour at room temperature. Sections were then stained with the universal immuno-enzyme polymer method, and developed with 3'3-diaminobenzidine hydrochloride using a Histofine Sample Stain Max Po kit (Nichirei Co., Tokyo). The cell proliferation index was calculated from the five fields in regions of five rats with the highest concentration of Ki-67-positive nuclei in each group examined under a high power microscope $(\times 400)$. The labeling index was determined ${ }^{18)}$ as the percentage of positive cells using ImageJ software and the mean was calculated.

The tumor volume was measured in each sacrificed rat. After H\&E staining, images $(\times 40$ magnification) of control and hypoxic sections were taken under a light microscope (Olympus, Osaka). A 1-mm slide scale image was also observed at the same magnification. Measurements were performed with ImageJ software. The tumor volume $\left(\mathrm{mm}^{3}\right)$ was defined as $1 / 2 a b^{2}$ (a: long diameter, b: short diameter), as previously described (Fig. 2). ${ }^{5,21)}$

Differences in tumor volume between the groups were analyzed using the t-test (Sigma Plot software; Systat Software Inc., San Jose, Calif., U.S.A.). Data are presented as mean \pm standard error.
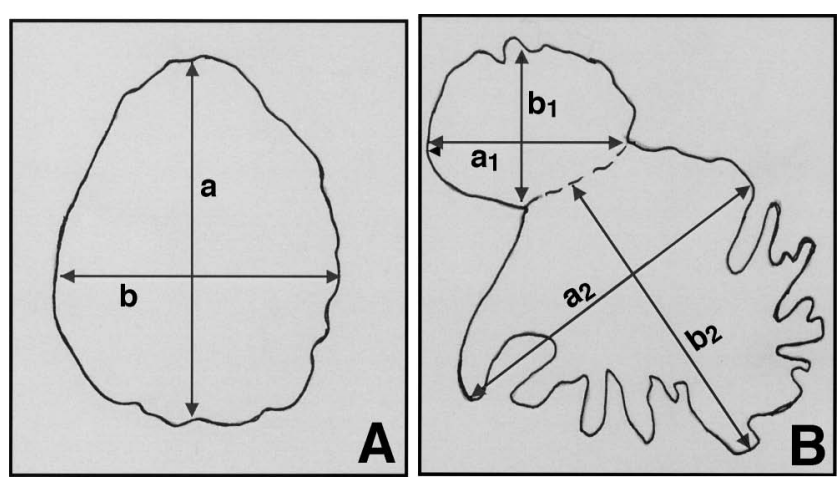

Fig. 2 Method for measuring the volume of the tumor. The volume was defined as $1 / 2 \mathbf{a b}^{2}$ (a: long diameter, b: short diameter). A: Regular tumor volume was simply calculated by the formula. B: Irregular tumor volume was calculated as the total of each part of the lesion $1 / 2 \quad a_{1} b_{1}{ }^{2}+1 / 2 \quad a_{2} b_{2}{ }^{2}$. Necrotic areas containing tumor cells were included in the tumor volume.

\section{Results}

All 34 rats tolerated the injection of the glioma cells. All rats in the hypoxia group had developed neurological deficits on the day following occlusion, which gradually increased. No rats in the control group had neurological deficits or signs.

Histological examination of the brain sections revealed glioma formation in the control and hypoxia groups, and tumor pseudopalisading in the hypoxia group characterized by accumulation of tumor cells around the hypoxic area (Fig. 3). No pseudopalisading was found in the control group. Tumor pseudopalisading was observed near the ischemic area, with evidence of formation of new blood vessels (Fig. 4A-C). The tumor and the normal brain had a clear border in the control group (Fig. 4D).

The quantitative tumor volume measurements indicate significant differences between the control and hypoxia groups. Mean tumor volume in the hypoxia group $\left(10.5 \pm 0.59 \mathrm{~mm}^{3}\right)$ was significantly larger than that in the control group $(6.9 \pm 0.93$ $\left.\mathrm{mm}^{3}\right)(\mathrm{p}<0.005)$. However, only the tumor volume in the hypoxia group included necrotic areas adjacent to the pseudopalisading cells.

Immunohistochemical staining found Ki-67-positive nuclear staining in the control group and around the ischemic area in the hypoxia group (Fig. 5). The mean Ki-67 labeling index was $42 \%$ in the control group $(n=10)$ and $45 \%$ in the hypoxia group $(\mathrm{n}=10)$, with no significant difference, indicating that the glioblastoma tumor cells excluding 


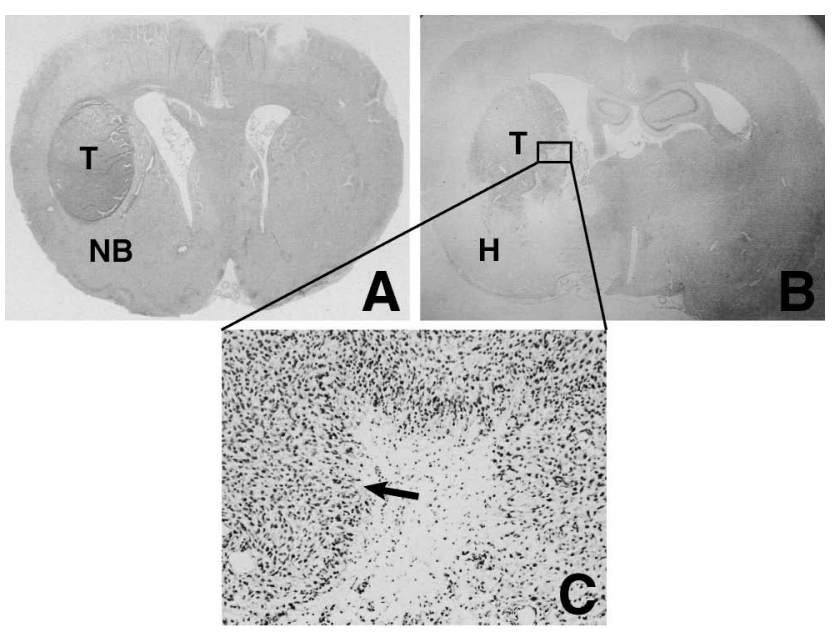

Fig. 3 A, B: Photographs of representative C6 glioma formation in a coronal slice of the control group (A) and the hypoxia group with peripheral brain ischemia (B) on day 10 . $\mathrm{H}$ : hypoxia, NB: normal brain, T: tumor. C: Photomicrograph of (B) showing pseudopalisading (arrow). Hematoxylin and eosin stain, (A) $\times 10$, (B) $\times 10$, (C) $\times 200$.

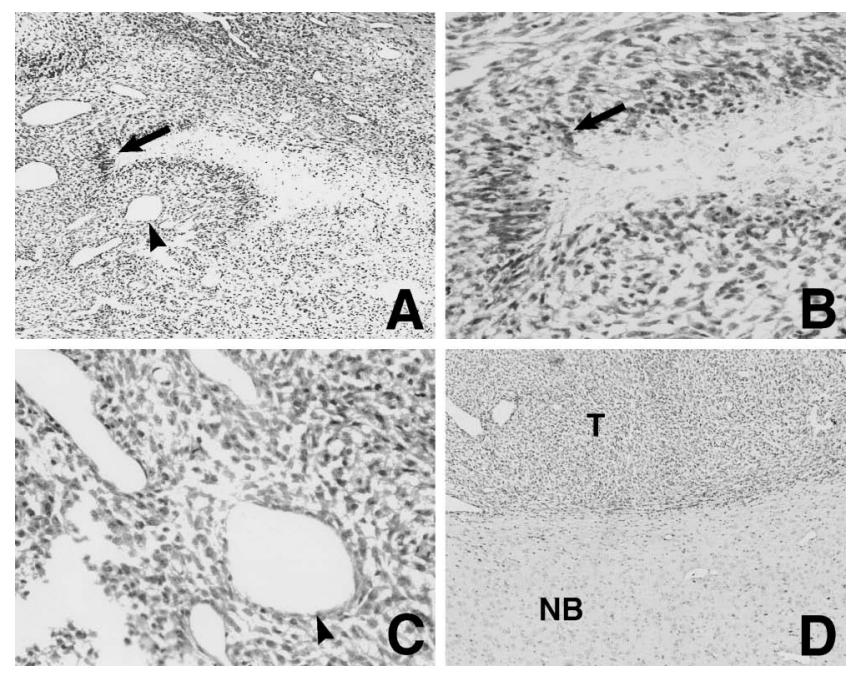

Fig. 4 A-C: Photomicrographs showing microvascular proliferation (arrowhead) and pseudopalisading (arrow) around the necrosis in the hypoxia group. D: Photomicrograph showing absence of pseudopalisading, and glial cells tightly arranged with a clear border between the tumor ( $T$ ) and the normal brain (NB) in the control group. Hematoxylin and eosin stain, (A) $\times 200$, (B) $\times 400$, (C) $\times 400$, (D) $\times 200$.

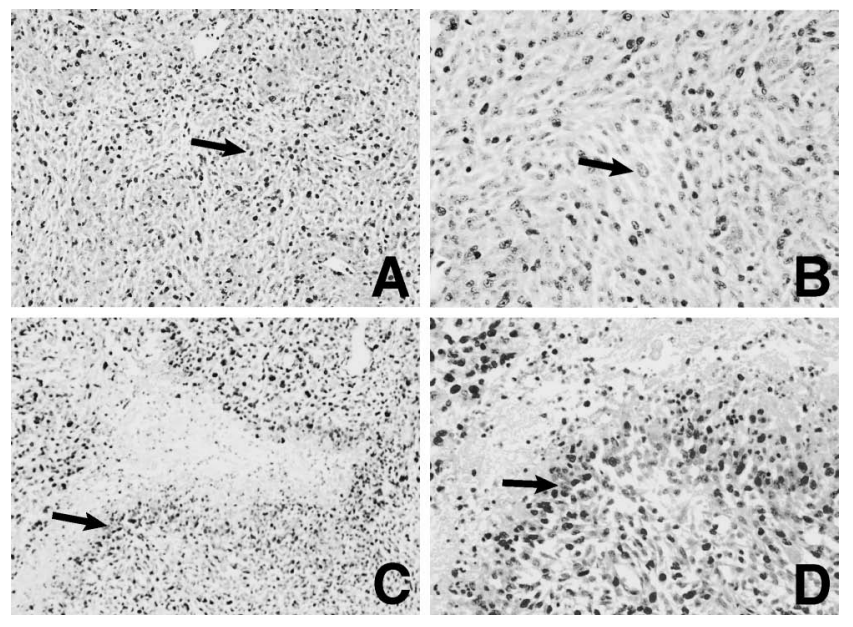

Fig. 5 A, B: Photomicrographs showing positive Ki-67 staining (arrow) in the nuclei of the tumor cells with mean labeling index of $\mathbf{4 2 \%}$ in the control group. C, D: Photomicrographs showing positive Ki-67 staining (arrow) in the nuclei of the tumor cells, but little staining in the necrotic region adjacent to the pseudopalisading in the hypoxia group, with mean labeling index of $45 \%$. (A) $\times 200$, (B) $\times 400$, (C) $\times 200$, (D) $\times 400$.

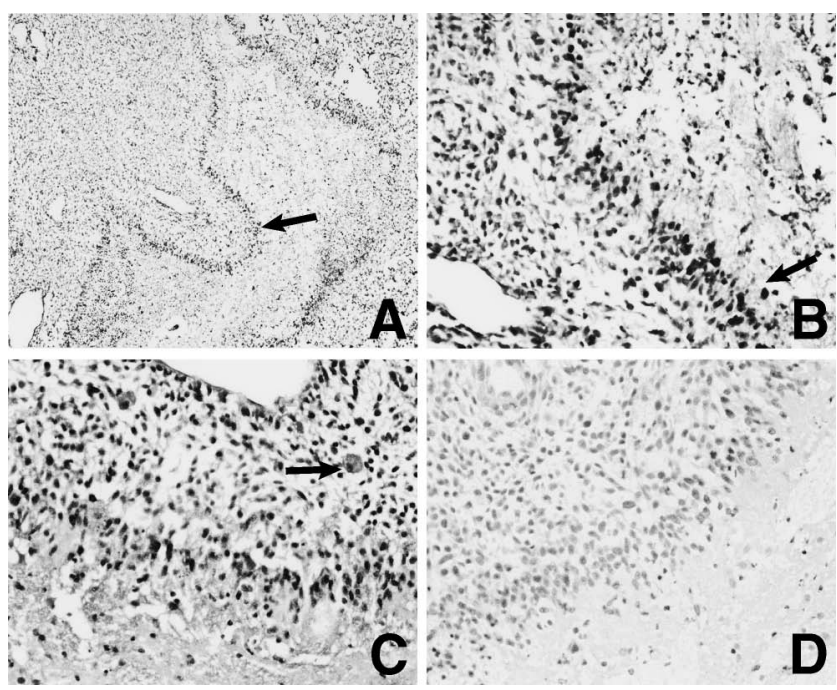

Fig. 6 Immunohistopathological examination for hypoxia-inducible factor (HIF)- $1 \alpha$ and vascular endothelial growth factor (VEGF) expression in the hypoxia group. A, B: Photomicrographs showing intense nuclear HIF-1 $\alpha$ immunoreactivity in pseudopalisading cells (arrow) around areas of necrosis. (A) $\times 200$, (B) $\times 400$. C, D: Photomicrographs showing VEGF immunoreactivity in the cytoplasm (arrow) of pseudopalisading cells around the necrosis $(C: \times 400)$, and negative control for VEGF staining (D: $\times 400)$. 
necrosis had the same proliferative potential in both groups.

Immunohistochemistry showed clear nuclear staining for HIF- $1 \alpha$ in the pseudopalisading cells around the necrosis area, and cytoplasmic staining for VEGF in the pseudopalisading cells, but not in the control group (Fig. 6).

\section{Discussion}

The present study induced experimental brain hypoxia by PMCAO in combination with the implantation of gliomas in rats. However, we need to clarify the meaning of the brain hypoxia. This study could not confirm that the glioma cells were more hypoxic in the hypoxia group than in the control group. The entire hemisphere containing the tumor affected by the PMCAO is really more hypoxic than the corresponding hemisphere in the control group.

Similar PMCAO models without tumors have shown that HIF- $1 \alpha$ induces angiogenesis. ${ }^{16)}$ HIF- $1 \alpha$ is a transcription factor that regulates the adaptive response to hypoxia in mammalian cells, and after cerebral ischemia, hypoxia-induced VEGF expression precedes neovascularization. ${ }^{19)}$ Brain tissue within the penumbra of cerebral ischemia becomes hypoxic and responds with increased VEGF expression mediated via the transcription factors HIF-1 and HIF-2. Subsequently, both VEGF-1 and VEGF-2 expression are induced, and finally angiogenic reaction occurs, characterized by proliferating endothelial cells and neovascularization.6,9)

In the present study, the effect of such angiogenic reaction under hypoxic conditions was reflected in slightly larger tumor volume in the hypoxia group compared to the control group. However, the tumor volume in each group cannot be simply compared, since the hypoxic condition had caused widespread and scattered ischemia in the brain, thus allowing space for the tumor volume to increase. Moreover, the glioma volume in the hypoxia group was calculated as the total volume including the pseudopalisading necrosis. The present study did establish that hypoxia did not reduce the tumor volume in the ischemic brain. Glioma growth labeling index, Ki-67, showed no significant difference between the two groups, suggesting that ischemia did not affect cell proliferation in vivo. The most interesting findings were the dynamic pathological changes in the hypoxic glioma model. The most prominent histological finding in the hypoxia group was abundant formation of pseudopalisading around the necrosis in the tumor. The necroses consisted of multiple small irregular band-like or serpiginous foci, surrounded by radially orientated, densely packed, small fusiform glioma cells in a 'pseudopalisading' pattern, a histological characteristic of glioblastoma. ${ }^{14)}$

Fas/Apo-1 (CD95), an apoptosis-mediating cell membrane protein, is one of the preferentially expressed molecules surrounding necrosis in glioma cells. Although the Fas families are widely involved in the necrogenesis of glioblastoma, this molecule is not always induced by hypoxic conditions. ${ }^{20)}$ The following hypothesis has been proposed to account for the presence of necrogenesis with pseudopalisading in glioblastoma. ${ }^{3,17)}$ Vascular occlusion and intravascular thrombosis lead to tissue hypoxia in the perivascular region. The tumor cells then become hypoxic and undergo apoptosis or necrosis, eventually leaving a central necrotic zone. Vessels distant to the vascular occlusion become necrotic and degenerate. ${ }^{4)}$ Hypoxic pseudopalisading cells show increased HIF-mediated transcription and VEGF secretion, leading to local microvascular hyperplasia. ${ }^{3)}$ The present study found little vascular occlusion in the small vessels of our hypoxic model, and the final development of pseudopalisading necrosis was a typical histological finding. Therefore, we conclude that induced hypoxia caused pseudopalisading necrosis around the implanted C6 glioma cells in the experimental glioblastoma rat model.

\section{References}

1) Acker T, Plate $\mathrm{KH}$ : A role for hypoxia and hypoxiainducible transcription factors in tumor physiology. J Mol Med 80: 562-575, 2002

2) Benda P, Someda K, Messer J, Sweet WH: Morphological and immunochemical studies of rat glial tumors and clonal strains propagated in culture. J Neurosurg 34: 310-323, 1971

3) Brat DJ, Castellano-Sanchez AA, Hunter SB, Pecot M, Cohen C, Hammond EH, Devi SN, Kaur B, Van Meir EG: Pseudopalisades in glioblastoma are hypoxic, express extra cellular matrix proteases and are formed by an actively migrating cell population. Cancer Res 64: 920-927, 2004

4) Brat DJ, Van Meir EG: Vaso-occlusive and prothrombotic mechanisms associated with tumor hypoxia, necrosis, and accelerated growth in glioblastoma. Laboratory Invest 84: 397-405, 2004

5) Carlsson G, Gullberg B, Hafstrom L: Estimation of liver tumor volume using different formulas - an experimental study in rats. J Cancer Res Clin Oncol 105: 20-23, 1983

6) Damert A, Machein M, Breier G, Fujita MQ, Hanahan D, Risau W, Plate KH: Up-regulation of vascular endothelial growth factor expression in a rat glioma is conferred by two distinct hypoxia-driven mechanisms. Cancer Res 57: 3860-3864, 1997 
7) George P, Charles W: The Rat Brain in Stereotaxic Coordinates, ed 3. Sydney, Australia, Academic Press, 1997

8) Gonzalez-Falcon A, Candelario-Jalil E, GarciaCabrera M, Leon OS: Effects of pyruvate administration on infarct volume and neurological deficits following permanent focal cerebral ischemia in rats. Brain Res 990: 1-7, 2003

9) Gratas C, Tohma Y, Barnas C, Taniere P, Hainaut P, Ohgaki H: Up-regulation of Fas (APO-1/CD95) ligand and down-regulation of Fas expression in human esophageal cancer. Cancer Res 58: 2057-2062, 1998

10) Grobben B, De Deyn PP, Slegers H: Rat C6 glioma as experimental model system for the study of glioblastoma growth and invasion. Cell Tissue Res 310: 257-270, 2002

11) Harris AL: Hypoxia - A key regulatory factor in tumor growth. Nat Rev Cancer 2: 38-47, 2002

12) Kaur B, Khwaja FW, Severson EA, Matheny SL, Brat DJ, Van Meir EG: Hypoxia and the hypoxia-inducible-factor pathway in glioma growth and angiogenesis. Neuro Oncol 7: 134-153, 2005

13) Koizumi J, Yoshida Y, Nakazawa T, Ooneda G: Experimental studies of ischemic brain edema. A new experimental model of cerebral embolism in rats in which recirculation can be introduced in the ischemic area. Jpn J Stroke 8: 1-8, 1986

14) Lantos PL, VandenBerg SR, Kleihues P: Tumors of the nervous system, in Graham DI, Lantos PL (eds): Greenfield's Neuropathology. London, Arnold, 1996, pp 583-879

15) Marti HJ, Bernaudin M, Bellail A, Schoch H, Euler M, Petit E, Risau W: Hypoxia-induced vascular endothelial growth factor expression precedes neovascularization after cerebral ischemia. Am J Pathol 156: 965-976, 2000

16) Matsuda T, Abe T, Wu JL, Fujiki M, Kobayashi $H$ : Hypoxia-inducible factor-1alpha DNA induced angiogenesis in a rat cerebral ischemia model. Neurol Res 27: 503-508, 2005

17) Rong Y, Post DE, Pieper RO, Durden DL, Van Meir EG, Brat DJ: PTEN and hypoxia regulate tissue factor expression and plasma coagulation by glioblastoma. Cancer Res 65: 1406-1413, 2005

18) Sho A, Kondo S, Kamitani H, Otake M, Watanabe T: Establishment of experimental glioma models at the intrinsic brainstem region of the rats. Neurol Res 29: 36-42, 2007

19) Sun Y, Jin K, Xie L, Childs J, Mao XO, Logvinova A, Greenberg DA: VEGF-induced neuroprotection, neurogenesis and angiogenesis after focal cerebral ischemia. J Clin Invest 111: 1843-1851, 2003

20) Tohma Y, Gratas C, Van Meir EG, Desbaillets I, Tenan $M$, Tachibana O, Kleihues P, Ohgaki H: Necrogenesis and Fas/APO-1 (CD95) expression in primary (de novo) and secondary glioblastomas. J Neuropathol Exp Neurol 57: 239-245, 1998
21) Watanabe $K$, Sakamoto $M$, Somiya $M$, Amin $M R$, Kamitani H, Watanabe T: Feasibility and limitations of the rat model by $\mathrm{C} 6$ gliomas implanted at the subcutaneous region. Neurol Res 24: 485-490, 2002

22) Zagzag D, Lukyanov Y, Lan L, Ali MA, Esencay M, Mendez O, Yee H, Voura EB, Newcomb EW: Hypoxia-inducible factor 1 and VEGF up regulate CXCR4 in glioblastoma: implications for angiogenesis and glioma cell invasion. Lab Invest 86: 1221-1232, 2006

23) Zagzag D, Zhong H, Scalzitti JM, Laughner E, Simons JW, Semenza GL: Expression of hypoxia-inducible factor 1alpha in brain tumors: association with angiogenesis, invasion, and progression. Cancer 88: 2606-2618, 2000

Address reprint requests to: Hideki Kamitani, M.D., Department of Neurosurgery, Tottori Prefectural Kousei Hospital, 150 Higashishouwa-machi, Kurayoshi, Tottori 682-0804, Japan. e-mail: kamitanih@pref.tottori.jp

\section{Commentary}

The authors have performed an interesting study in which they implanted the C6 glioma cell line into the rat brain, and then occluded the middle cerebral artery ipsilateral to the tumor. They observed larger tumors with several histopathological changes when compared to controls. The histopathological changes in the middle cerebral artery occluded rats included an increased degree of pseudopalisading with upregulation of HIF1alpha and VEGF.

Pseudopalisading has always been a histopathological curiosity in glioblastoma. To my knowledge, no other tumor in humans can demonstrate such a histopathological alteration. In this study, the authors have demonstrated that HIF1alpha and VEGF may play a role in the subsequent growth of the tumor in the ischemic hemisphere, and may also account for the pseudopalisading that is found.

If hypoxia stimulates the growth of tumor cells in this way, then it poses the question of how can we avoid hypoxia in human tumors so as to prevent pseudopalisading and increased brain tumor growth. No doubt this will be a fruitful field of inquiry for the authors in years to come. I congratulate them on their nice study.

James T. RUTKA, M.D., F.R.C.S.C., F.A.C.S., F.A.A.P. Dan Family Chair in Neurosurgery Division of Neurosurgery The University of Toronto Toronto, Ontario, Canada 